\title{
Fuzzy Spatial Growing for Glioblastoma Multiforme Segmentation on Brain Magnetic Resonance Imaging ${ }^{\star}$
}

\author{
Alejandro Veloz ${ }^{1}$, Steren Chabert ${ }^{1}$, Rodrigo Salas ${ }^{1}$, Antonio Orellana ${ }^{2}$, \\ and Juan Vielma ${ }^{3}$ \\ ${ }^{1}$ Departamento de Ingeniería Biomédica, Universidad de Valparaíso, Chile \\ alejandro.veloz@yahoo.es, steren.chabert@uv.cl, rodrigo.salas@uv.cl \\ 2 Servicio de Neurocirugía, Hospital Carlos Van Buren, Valparaíso, Chile \\ ${ }^{3}$ Servicio de Imagenología Compleja, Hospital Carlos Van Buren, Valparaíso, Chile
}

\begin{abstract}
Image segmentation is a fundamental technique in medical applications. For example, the extraction of biometrical parameter of tumors is of paramount importance both for clinical practice and for clinical studies that evaluate new brain tumor therapies.

Tumor segmentation from brain Magnetic Resonance Images (MRI) is a difficult task due to strong signal heterogeneities and weak contrast at the boundary delimitation. In this work we propose a new framework to segment the Glioblastoma Multiforme (GBM) from brain MRI. The proposed algorithm was constructed based on two well known techniques: Region Growing and Fuzzy C-Means. Furthermore, it considers the intricate nature of the GBM in MRI and incorporates a fuzzy formulation of Region Growing with an automatic initialization of the seed points.

We report the performance results of our segmentation framework on brain MRI obtained from patients of the chilean Carlos Van Buren Hospital and we compare the results with Region Growing and the classic Fuzzy C-Means approaches.
\end{abstract}

Keywords: Fuzzy Spatial Growing (FSG), Magnetic Resonance Imaging (MRI), Glioblastoma Multiforme, Fuzzy C-Means, Region Growing, Anisotropic Diffusion Filter, Image Segmentation.

\section{Introduction}

The high definition, contrast and resolution of soft tissues obtained with Magnetic Resonance Imaging (MRI), makes this image modality very useful in the characterization of many pathological diseases located at the Central Nervous System (CNS) (see [8, [9] and [10]). Image processing techniques, such as segmentation, have motivated the development of many quantitative analysis methods to improve diagnostic and therapeutical outcomes (see [1, [9] and [15]).

\footnotetext{
* This work was supported by Research Grant Fondecyt 11060036, 1061201 and in part by the international cooperation Fondecyt Grant 7070262.
} 
In the present work, our interest is advocated to Glioblastoma Multiforme (GBM) segmentation from MRI. This class of glial tumor (also called glioma grade IV) has the highest mortality and morbidity ratio between all known brain tumors, due primarily to its very aggressive pathological behavior. This aggressive behavior causes uncertainty in the pathological border definition and constitutes the main inconvenient to obtain a precise anatomical diagnosis (see [4] and [10]).

In the context of medical images many different segmentation methods exist, but there is no universally applicable segmentation technique for all kind of image characteristics even for the same acquisition modality (see [6]). To develop or to apply a segmentation method, the specific pathology characteristics must be considered before analyzing the image (see [1] and [13]).

An improved framework to segment the Glioblastoma Multiforme is proposed. This method considers the fuzzy nature of the pathological appreciation made by radiologist. This new algorithm incorporates the fuzziness definition of the GBM boundaries in MRI. The formulation of our method is based on the classical Region Growing and Fuzzy C-Means algorithms to perform tumor segmentation, and considers the pathological nature of the GBM. We call our algorithm Fuzzy Spatial Growing (FSG) for GBM segmentation.

We report the performance results of our segmentation framework on brain MRI obtained from patients of the chilean Carlos Van Buren Hospital and a comparative study with the classical Region Growing and Fuzzy C-Means algorithms is made.

This work is organized as follows. In section 2 the proposed segmentation framework is stated. Section 3 shows the results obtained by applying the segmentation framework to the dataset. Discussion of the results are given in section 4. Finally concluding remarks are given in the last section.

\section{Methodology}

In this section we propose a framework for the GBM segmentation on MRI. We begin the explanation with the description of the available images and then the segmentation algorithms.

\subsection{Magnetic Resonance Images Dataset}

The images available for this study were obtained from two patients of the Carlos Van Buren chilean Hospital. The patients were histopathologically confirmed to be affected with Glioblastoma Multiforme. The images were acquired in a $1.5 \mathrm{~T}$ General Electric (GEMS, Milwaukee, USA) MRI System1 1 . Each slice consists of the following featured images: in the axial plane, T1-weighted (Fluid Attenuated Inversion Recovery (FLAIR) sequence, with TE/TR/TI of 24/1875/750 ms),

${ }^{1}$ This study has the corresponding agreement and authorization of the Carlos Van Buren Hospital. 
T1-weighted + C (with gadolinium contrast enhancement, Spin Echo (SE) sequence, with TE/TR of 100/4375 ms and $80^{\circ}$ flip angle) and T2-weighted (Fast Spin Echo (FSE) sequence, with TE/TR of 100/4375 ms). In the coronal plane, T1-weighted $+\mathrm{C}$ and T2-weighted with the same acquisition parameters as described above. Lastly, in the sagittal plane, T1-weighted $+\mathrm{C}$ and T1-weighted were acquired. From the two patients we obtained 77 images from a total of 32 anatomical slices, with spatial resolution of $4 \mathrm{~mm}^{3}$ and a slice gap of $1.5 \mathrm{~mm}$.

\subsection{Anisotropic Diffusion Filter}

A fundamental step in medical image processing is the application of filters to minimize effect of noise. Classical techniques for noise reduction, such as gaussian filter or isotropic linear diffusion filter, shift the edges localization and blur the images. This situation is undesirable due to the mismatching between the original and resulting localization of the boundaries between regions (i.e. Glioblastoma Multiforme).

In this work, the anisotropic diffusion filter (see 2] and 7]) was employed to minimize noise contamination as well as to avoid boundary localization problems (i.e. blurring) by reduction of the diffusivity in the edges that have high gradient magnitudes. Their nonlinear process behaves as a feedback system that preserves the edges in the different regions by adapting a diffusivity function to the image gradient. The filter is given by:

$$
\frac{\partial f}{\partial t}=\operatorname{div}(g(f, t) \cdot|\nabla f|)
$$

where $|\nabla f|$ is the gradient magnitude of the image $f$, and $g(f, t)$ is the diffusivity function given by

$$
g(f, t)=\exp \left(-\frac{|\nabla f|^{2}}{\kappa^{2}}\right)
$$

where $\kappa$ is the diffusivity parameter and determines the gradients magnitudes where the diffusion will begin to decrease, and therefore the edges that will be preserved. The performance of anisotropic diffusion filter is strongly dependent on the diffusivity function $g(f, t)$ and on the time interval $t$ (or the number of iterations in the discrete domain). This function modulates the gradient magnitude in each iteration to decrease diffusion along the image prominent edges.

\subsection{Region Growing}

The Region Growing algorithm is a classical region-based approach for medical image segmentation (see 3 and [16]). The basic approach is to start with a set $n$ of seed points $v_{i}, i=1 . . n$, of voxels interactively selected. From these seed points regions grow by adding to each seed those neighboring voxels that have similar properties based on predefined criteria. In this successive growing process $n$ regions $R_{i}, i=1 . . n$, will be formed.

The similarity criteria to consider a voxel as member of the region $R_{i}$ is established according to the image properties, for example texture, topology, 
etc. In our implementation, let $x$ be a neighbor voxel to some voxel belonging to the region $R_{i}$. If the euclidean distance between the voxel $x$ and the seed point $v_{i}$ is less than a threshold $\theta$ then the voxel $x$ is included to the region $R_{i}$. Finally, the region of interest is obtained by merging each grown region $\bigcup_{i=1 . . n} R_{i}$.

Unfortunately, the performance of this algorithm will strongly depend on the correct selection of seed points and this selection depends directly on the histopathological behavior of the Glioblastoma Multiforme in MRI. The capability of the user (radiologist) to identify the several tumor domains will be of paramount importance for the appropriate growing process. Such domains will be, for example, necrosis, i.e., high cellularity activity, and other biological features proper to this tumor class.

\subsection{Fuzzy C-Means}

Other common approach to segment MR images is Fuzzy C-Means (FCM). This technique is an unsupervised clustering algorithm that has been extensively used in Pattern Recognition (see [14]). The fuzzy set obtained from classification of the intensity distribution is especially interesting in MRI of Glioblastoma Multiforme, due to the fuzzy definition between the tumor boundary and its surrounding brain tissue.

This unsupervised method is an iterative procedure of fuzzy partition of the $p$ dimensional feature space in $C$ clusters. The algorithm starts from $C$ randomly selected vectors $\left\{v_{1}, \ldots, v_{C}\right\}$ called centroids or clusters centers. The membership of each data $x_{j}, j=1 . . N$, to the class $i, i=1 . . C$, is computed with the membership function given by

$$
\mu\left(v_{i}, x_{j}\right)=\mu_{i j}=\frac{1}{\sum_{k=1}^{C}\left(\frac{d\left(x_{j}, v_{i}\right)}{d\left(x_{j}, v_{k}\right)}\right)^{\frac{2}{m-1}}}
$$

where $m$ is the fuzziness exponent and $d\left(x_{j}, v_{i}\right)$ is the euclidean distance measure between the $x_{j}$ feature vector and the $v_{i}$ centroid. The membership function (3) satisfies the properties that $0 \leq \mu_{i j} \leq 1$ and $\sum_{i=1}^{C} \mu_{i j}=1$. The centroids of each cluster are computed in each iteration as:

$$
v_{i}=\frac{\sum_{j=1}^{N} \mu_{i j}^{m} x_{j}}{\sum_{j=1}^{N} \mu_{i j}^{m}} \quad i=1 . . C
$$

The cost function, that should be minimized, is given by

$$
J_{m}=\sum_{j=1}^{N} \sum_{i=1}^{C} \mu_{i j}^{m} d^{2}\left(x_{j}, v_{i}\right)
$$

The centroids and the membership degrees of all features vectors are updated until there is no meaningful change in the cost function, or equivalently, in the centroid location in the feature space. 
In our application, we selected four clusters: fat/tumor, white matter, gray matter and cerebral spinal fluid (CSF)/background. Additionally, to improve the tumor classification with this method, a preprocessing (simply thresholding) step is applied in order to eliminate fat in the images.

After applying FCM a defuzzification stage is performed in order to convert the fuzzy membership of the feature vectors into a crisp set. This stage consists in the specification of a certain threshold for decoding the membership degrees into a crisp set to obtain the tumor voxels.

\subsection{Fuzzy Spatial Growing for Glioblastoma Multiforme Segmentation}

A new segmentation framework is introduced in this section to avoid the drawbacks of the above mentioned algorithms for Glioblastoma Multiforme segmentation. Our method is based on the classical Fuzzy C-Means and Seeded Region Growing approaches described above. We call our proposed framework Fuzzy Spatial Growing (FSG) for Glioblastoma Multiforme segmentation.

The FSG method incorporates an automatic procedure to obtain seed points. The method starts by applying first the anisotropic diffusion filter and then the Fuzzy C-Means (FCM) algorithm. The clusters are defuzzified and the tumor cluster is obtained. The tumor intensity features is obtained according to the skeleton of the tumor region obtained with FCM.

The skeleton is a mathematical morphology technique described in $[5]$. The skeleton determines the closest boundary points for each point in an object and allows to extract a region-based features representing the tumor of interest. With this approach, we obtain a vector composed by the histopathological intensity patterns across the tumor region represented by all tumor intensities in the skeleton (i.e., low to high contrast enhancement). The automatic seed points initialization is done on points located at the skeleton of the tumor (see figure1).

The membership function of the tumor voxels is constructed from the information of the skeleton intensity pattern and the filtered image is considered as a fuzzy set $F$. The membership values $\mu_{F}(x)$ are computed for each voxel $x$ of the image and depends on the information of the global gray-level image histogram. The membership function is a trapezoidal linguistic variable constructed as

$$
\mu_{F}(x)= \begin{cases}0 & 0 \leq x<\alpha \\ \frac{x-\alpha}{\beta-\alpha} & \alpha \leq x \leq \beta \\ 1 & \beta<x \leq \gamma \\ \frac{x-1}{\gamma-1} & \gamma<x \leq 1\end{cases}
$$

where the parameters $\alpha, \beta$ and $\gamma$ are computed from the histogram. $\alpha$ is the mode of the histogram and correspond to the most frequent intensities values of the intracraneal cavity. The parameters $\beta$ and $\gamma$ are the minimum and maximum of the tumor skeleton intensities respectively, and the parameters correspond to the range of intensities where the membership to the tumor is 1 , because we know for certain that the tumor has this intensities. The intrinsic heterogeneity signal of 
the GBM in MRI and the variable degree of GBM neighboring tissue infiltration determines that some intensities of poor contrast enhancement are between the histogram mode and $\beta$, that are formed mostly by the gray and white matter. Figure 1 shows how the gold standard has similar intensities than the gray and white matter. For this reason we define $\alpha$ as the mode of the histogram. On the other hand, some high cellularity tumor domains, blood vessels or fat have similar high intensities bigger than $\gamma$, then the voxels are considered to have some membership degree (less than one) at high intensity levels. The left side of figure 1 shows the membership function.

After the image fuzzy set $F$ is obtained, the spatial growing process begins from a seed extracted from the skeleton. All the skeleton voxels are included to the fuzzy tumor region $R$. The algorithm picks a voxel $x$ from $R$ and will add to the fuzzy tumor region $R$ all the neighboring voxels $v \in N_{8}(x)$ that satisfy the following similarity criteria:

$$
T\left\{\mu_{F}(x), S^{*}\left\{v_{1}, v_{2}, \ldots, v_{8}\right\}\right\} \geq \lambda
$$

where

$$
\begin{aligned}
S^{*}\left\{v_{1}, v_{2}, \ldots, v_{8}\right\}= & S\left\{S\left\{S\left\{\mu_{F}\left(v_{1}\right), \mu_{F}\left(v_{2}\right)\right\}, S\left\{\mu_{F}\left(v_{3}\right), \mu_{F}\left(v_{4}\right)\right\}\right\},\right. \\
& \left.S\left\{S\left\{\mu_{F}\left(v_{5}\right), \mu_{F}\left(v_{6}\right)\right\}, S\left\{\mu_{F}\left(v_{7}\right), \mu_{F}\left(v_{8}\right)\right\}\right\}\right\}
\end{aligned}
$$

$\lambda$ is an inclusion threshold, and, $T$ and $S$ are the T-norm and T-conorm respectively, examples of this triangular norms are the Zadeh and Lukasiewicz approaches (see [12]).
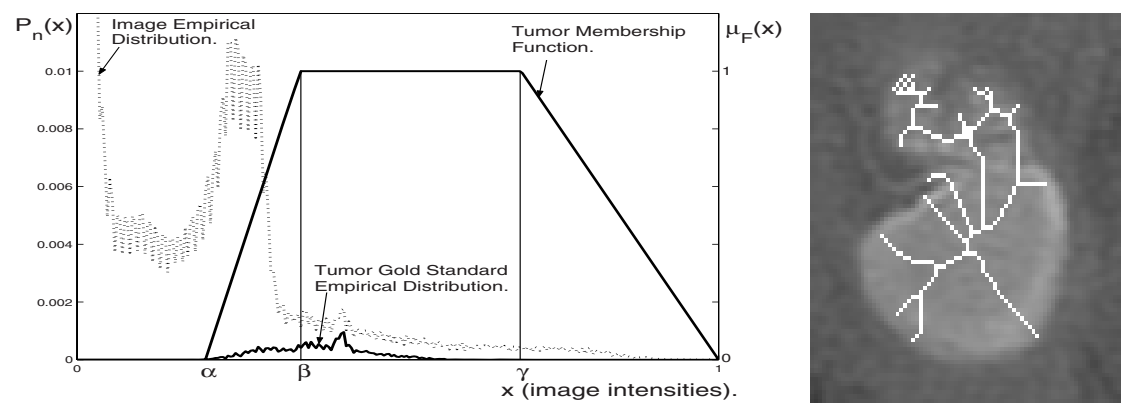

Fig. 1. (left) Histogram of the GBM MR image intensities, shows the empirical distribution of the intensities of the overall image and the tumor gold standard, furthermore it shows the tumor membership function used in the FSG algorithm. (right) Skeleton used to estimate the parameters of the tumor membership function.

\subsection{Evaluation Criteria}

To evaluate the quality of segmentation of the algorithms, GBM were manually segmented on MRI with the assistance of a neuroradiologist of the Carlos Van 
Buren Hospital. This manually segmented image will be our gold standard, or also called ground truth (see 9]).

The quality was evaluated with the accuracy index. The accuracy index is expressed as the percentage of tumor area present in both the segmented image and in the gold standard.

The false positive error $(F P)$ is expressed as the percentage of tumor area segmented that does not belong to the gold standard, i.e., region that was erroneously segmented as tumor region with the algorithm. The false negative error $(F N)$ is the percentage area of the tumor region of the gold standard that were not segmented with the algorithm.

\section{Results}

In this section, we compare the quality of segmentation of the Fuzzy Spatial Growing algorithm with the classical region growing and Fuzzy C-Means approaches. The Region Growing, FCM monospectral (1-dimensional) and FSG algorithms were applied to T1-weighted $+\mathrm{C}$ images. The FCM multispectral (2 and 3-dimensional) was applied to T1-weighted, T1-weighted $+\mathrm{C}$ and T2weighted images in the axial plane, T1-weighted, T1-weighted $+\mathrm{C}$ in coronal plane, and in T2-weighted and T1-weighted + C in sagittal plane. Additionally, for the FCM multispectral algorithm we present the results obtained with a preprocessing stage to eliminate the fat available in all the images.

The anisotropic diffusion filter was used with parameter $\kappa=10$ and with 10 iterations. In fuzzy clustering techniques a value of $m=2$ was employed for fuzziness exponent and four clusters were considered (gray matter, white matter, CSF-background and fat-tumor). Furthermore, all thresholds were applied interactively.

During the experiments, the FSG algorithm outperforms the Region Growing and FCM algorithms when the pathological condition of the tumor have an insufficient contrast enhancement and high fuzziness in the boundary between the tumor and the white matter. This phenomenon is due to the low cellular metabolism or high infiltration to the neighboring tissue. Figure 2 shows two cases, a typical segmented image and the worst GBM segmentation case.

The accuracy, false positive error and false negative error results of the three algorithms are shown in table 1. Note that our FSG algorithm outperforms in the three criteria to the other algorithms. This results will be discussed in the next section.

Table 1. Outcomes obtained with each segmentation method

\begin{tabular}{|c|c|c|c|}
\hline Technique. & Accuracy (std. desv.). & FP (std. desv.). & FN (std. desv.). \\
\hline Fuzzy Spatial Growing & $96.38 \%(7.16)$ & $9.18 \%(9.84)$ & $3.63 \%(7.16)$ \\
\hline Region Growing & $95.54 \%(7.35)$ & $7.65 \%(9.55)$ & $4.87 \%(7.64)$ \\
\hline FCM monospectral & $93.75 \%(12.63)$ & $5.51 \%(7.19)$ & $6.24 \%(12.62)$ \\
\hline FCM multispectral & $93.93 \%(12.36)$ & $5.78 \%(7.79)$ & $6.08 \%(12.35)$ \\
\hline FCM multispectral (fat eliminated) & $94.79 \%(9.25)$ & $5.63 \%(7.16)$ & $5.21 \%(9.25)$ \\
\hline
\end{tabular}



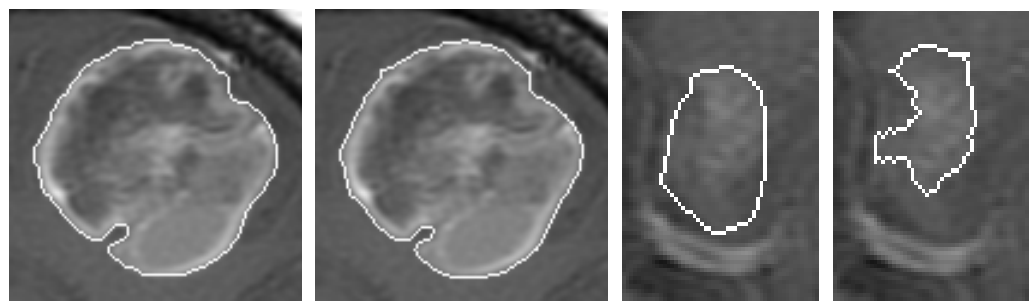

Fig. 2. Segmented T1-weighted + C Magnetic Resonance images of GBM with Fuzzy Spatial Growing algorithm: (left) Gold Standard of a segmented GBM. (middle-left) GBM segmented with FSG. (middle-right) Gold Standard of a segmented GBM, worst case. (right) Worst segmented case of the GBM with the FSG.

\section{Discussion}

We have described a flexible method for Glioblastoma Multiforme Segmentation in MRI that allows us to deal with the difficult properties of this tumor class. The difficulty is due to the aggressive infiltration of cancerous cells into neighboring tissue. This situation is the main disadvantage of the segmentation of this tumor and is the focus of our formulation.

The possible guidance of the user expert knowledge gives Fuzzy Spatial Growing approach an additional suitability and flexibility in the segmentation process and allows a continuous refinement of the outcomes.

The tumor segmentation result will depend on both the histopathological properties of the Glioblastoma Multiforme and the characteristics revealed in the MR image. After processing of all the images by Fuzzy Spatial Growing, Region Growing and FCM, these results were compared with the gold standard. We found both false positives and false negatives errors in the comparison between the segmented image and the gold standard. The false negative errors corresponds to areas with weak or intermediate contrast enhancement in the tumor boundary. In this sense, Fuzzy Spatial Growing was better to include these areas, but yet it could be further optimized.

In those GBM boundary areas between the tumor and neighboring tissue where the tumor presents high cellular activity pattern, the performance of the FCM (monospectral and multispectral), and Region Growing algorihtms were higher than $95 \%$. Instead, in situations where the tumor has low contrast enhancement areas the performance of these algorithms was poor in terms of accuracy, with respect to the tumor areas that have more angiogenesis ratio (formation of blood vessels, resulting in high intensities and better definition of the tumor). For this reason, the possibility to obtain a good performance of the segmentation algorithms resides in the detection of these tumor areas that exhibit low contrast enhancement.

Fuzzy Spatial Growing has the advantage that it does not require seed points initialization, i.e., the parameters selection by the users are minimized, although 
the FSG requires at least one seed point, automatically selected. On the other hand, the drawback of the Region Growing approach in this work is that the seed points should be selected manually by the users, and the performance of the algorithm will depend on the expert knowledge to identify the several tumor areas like necrosis, low and high contrast enhancement domains.

The FCM approach is intrinsically an automated method and we used it as automatic initialization of the seed points for the FSG. Furthermore, the intensity patterns obtained for both fuzzification stage and automatic seed points initialization take into account more information content along the tumor that the classical method of manually select seed points.

The assumptions that we made about the parameter values to obtain the fuzzy set of the tumor and to initialize a single seed point work well in all of our images. Furthermore, in the different stages of our method, the user can modify parameter values in order to consider certain biological situations of the GBM, that allows to refine and to grant flexibility of the segmentation process.

The only necessary user interaction consists in selecting a region of interest to specify the tumor location and the threshold $\lambda$ that controls the spatial growing of the obtained tumor border.

\section{Conclusion}

In this work, we introduced and applied a suitable algorithm to segment Glioblastoma Multiforme on Magnetic Resonance Images. Furthermore, our reliable method combines the expert knowledge and fuzzy properties of Glioblastoma Multiforme to segment the brain tumor slices separately.

Our developed algorithm is based on classical approaches for image segmentation, such as Fuzzy C-Means and Mathematical Morphology to extract pattern of intensities of the Glioblastoma Multiforme and to select seed points automatically to perform the Fuzzy Spatial Growing. Additionally, a Fuzzy similarity criteria is considered to measure the voxels memberships to the tumor.

Further work is needed to incorporate the bias field estimation to correct or compensate the intensity inhomogeneities introduced during the acquisition process in MRI. The FSG algorithm can be applied to other types of brain tumors, such as low grade gliomas. Last but not least, other types of membership functions to obtain the fuzzy sets can be explored in the algorithm.

In conclusion, Fuzzy Spatial Growing approach constitutes an applicable method to the daily clinical practice for Computer Assisted Techniques that have an enormous potential to increase the safety in surgical intervention of Glioblastoma Multiforme, improving the surgical outcome and the prognosis of the patients.

\section{Acknowledgements}

We would like to thank to the Imaging Service of the Carlos Van Buren Hospital for providing the access to the images used in this work. 


\section{References}

1. Gering, D., Nabavi, A., Kikinis, R., Hata, N., O'Donnell, L., Grimson, W., Jolesz, F., Black, P., Wells, W.: An integrated visualization system for surgical planning and guidance using image fusion and an open MR. Journal of Magnetic Resonance Imaging 13, 967-975 (2001)

2. Bloch, I., Gousseau, Y., Matre, H., Matignon, D., Pesquet-Popescu, B., Schmitt, F., Sigelle, M., Tupin, F.: Le traitement des images, Polycopié du cours ANIM, Département TSI - Télécom-París (2004)

3. Feng, D.: Segmentation of soft tissues in medical images, Ph.D. thesis, National University of Singapore (2005)

4. Gaensler, E.H.: Neurorradiología fundamental, Marban (1998)

5. González, R., Woods, R.: Digital image processing, 2nd edn. Prentice Hall, Englewood Cliffs (2001)

6. Bonnie, N.J., Melanie, B.F., Carolyn, C.M., Qing-shou, H., Roger, S.D., Phil, J.G., Michael, E.B.: Brain tumor volume measurement: Comparison of manual and semiautomated methods. Radiology 212, 811-816 (1999)

7. Jahne, B., Haubecker, H.: Computer vision and applications. Academic Press, London (2000)

8. Kalousek, M., Birbamer, G., Kampfl, A., Aichner, F., Felber, S.: Principal advantages of CNS diagnostics by magnetic resonance imaging. Neurolog/'ia cro/'atica 40(2), 73-84 (1991)

9. Kaus, M.: Contributions to the automated segmentation of brain tumors in magnetic resonance images, Ph.D. thesis, Der Technischen Fakultat der Universitat Erlangen-Nurnberg (1999)

10. Kaye, A.H., Laws, E.R.: Brain tumors, Churchill Livingstone Edition (1995)

11. Moon, N., Bullitt, E., Van Leemput, K., Gerig, G.: Model-based brain and tumor segmentation. In: IEEE International Conference on Pattern Recognition, pp. 528531. IEEE Press, Los Alamitos (2002)

12. Moraga, C., Salas, R.: A new aspect for the optimization of fuzzy if-then rules. In: Proceedings. 35th International Symposium on Multiple-Valued Logic, pp. 160 165. IEEE Press, Los Alamitos (2005)

13. Oppelt, A.: Imaging systems for medical diagnostics. Wiley, Chichester (2006)

14. Shen, S., Sandham, W., Granat, M., Sterr, A.: MRI fuzzy segmentation of brain tissue using neighborhood attraction with neural-network optimization. IEEE Transaction on Information Technology in Biomedicine 9(3), 459-467 (2005)

15. Veloz, A.: Esquema de trabajo para segmentar glioblastomas multiformes en MRI mediante técnicas de crecimiento de regiones y fuzzy c-means, Tesis de Grado de Ingeniería Biomédica, Universidad de Valparaíso (2007)

16. Xuan, J., Adah, T., Wang, Y.: Segmentation of magnetic resonance brain image: Integrating region growing and edge detection. In: IEEE Conference on Image Processing, pp. 544-547. IEEE Press, Los Alamitos (1995) 